\title{
DIAMOND DETECTORS FOR FAST TRANSIENT SCRAM MEASUREMENTS - THE DETECTOR DEVELOPMENT
}

\author{
Ondřej Novák ${ }^{a, *}$, Lubomír Sklenka $^{a}$, Michal Pomorski $^{b}$, \\ Clement LyNDE ${ }^{c}$
}

${ }^{a}$ Czech Technical University in Prague, Faculty of Nuclear Sciences and Physical Engineering, Department of Nuclear Reactors, V Holešovičkách 2, 18000 Prague 8, Czech Republic

${ }^{b}$ CEA (French Atomic Energy Comission), LIST, Diamond Sensors Laboratory, CEA Saclay, 91191 Gif-sur-Yvette CEDEX, France

${ }^{c}$ CEA (French Atomic Energy Comission), LIST, Sensors and Electronic Architectures Laboratory, CEA Saclay, 91191 Gif-sur-Yvette CEDEX, France

* corresponding author: ondrej.novak2@fjfi.cvut.cz

Abstract. This paper presents a development of special diamond detector for time dependent neutron flux measurements in a nuclear reactor. Neutron flux changes during SCRAM are difficult to measure by existing detector technologies. Therefore, a new detector is developed. This new type diamond based detector should allow to perform fast measurements of neutron flux during SCRAM with high pulse count rate. The diamond detector design and signal processing are described in this paper including the pulse shape discrimination method. In addition to theoretical detector study, three detector was manufactured at CEA Saclay. Experience gathered during manufacturing is described as well.

KEYWORDS: Diamond detector, neutron detection, nuclear reactor, pulse shape analysis, boron conversion, fast transient, SCRAM, VR-1 reactor.

\section{INTRODUCTION}

Reactor operation is connected with events that have direct impact on reactor operation and reactor nuclear safety. These events are commonly defined as postulated initiating events (PIEs) [1] and are used in deterministic safety theory. One area of PIE contains fast changes of reactivity (like RIA ${ }^{1}$ or SCRAM ${ }^{2}$. Those events initiate a fast transient process, where the neutron flux distribution and parameters are rapidly changed.

Deep and clear understanding of the SCRAM fast transient processes are highly challenging due to several reasons. They are complex, space dependent and mostly, they happen very fast. In addition, they have significant impact on the nuclear safety. For better understanding, the currently available experimental instrumentation should be improved (new, faster detectors and detecting systems) which will result in better reactor core monitoring, faster, and more accurate prediction of core behavior. This improvement in the field of fast transient experiments will conclude in enhancement of codes assigned for fast transient studies.

Precise study of the RIA or SCRAM flux distribution changes that are happening during the event have not been carried out due to lack of high performance neutron detectors [2, 3]. In other words, a new detection system must be developed. The final goal of this study is to measure neutron flux behavior during

\footnotetext{
${ }^{1}$ Reactivity Initiated Accident

${ }^{2}$ Emergency shutdown of a nuclear reactor
}

SCRAM. This means to:

(1.) develop fast performing detector (including electronic and data processing) that will be able to provide fast accurate measurement of neutron flux inside reactor core;

(2.) provide calculation tools that will be able to simulate the experiment proposed;

(3.) design and perform the SCRAM measurement (at the VR-1 reactor) with new detector developed;

(4.) analyze experimental and data calculated and conclude results.

This article presents the progress in the first step the detector development.

\section{DiAmond DETECTORS}

Current detectors available on the market are not fast enough to perform high accuracy short measurements for the rod SCRAM instrumentation. Due to this, new detector needs to be developed. To demonstrate capabilities requested from the new detector, the following example is presented: One case of fast transient measurement application is a study of the neutron flux changes during the fast control rod insertion (SCRAM). The control rods are usually inserted in few seconds. At the VR-1 reactor, SCRAM duration is less than $0.5 \mathrm{~s}$. During this very short time period, large amount of interaction needs to be recorded. If 500 time steps during SCRAM are requested, each step should contain more than one thousand thermal 
neutron interactions, in conclusion, more than one million neutron pulses per second should be recorded. However, the total number of interaction will be several times higher because of the gamma, electrons and fast neutrons, which will interact in the detector active volume as well. Based on the example presented, the experimental setup should be fast enough to collect and process more than ten million events per second. This puts demands on the detector, the amplifier and the analog digital (AD) converter.

Requirements stated are difficult to fulfill. Based on results from the first tests $[4]^{3}$, the detectors made of diamond crystals are highly promising (if this technology is developed and adjusted for this particular application). The diamond detectors contain a single crystal diamond that serves as active volume. The charge deposited by ionizing interactions is collected by an electrical field created by the applied voltage on two electrodes deposited on the surface. The diamond mono crystals have unique material properties and are very promising for the application in the detection of chemical compounds and radiation [5]. General overview of the diamond based detectors is summarized in [6]. The detectors offer variety of options in the crystal size, design and in the overall detector construction. Main advantages of the diamond detector are:

- Pulse length from the diamond detector is around $10 \mathrm{~ns}$. This very short duration results in almost no detector dead time and therefore theoretically, the detector yield can reach up to $10^{8}$ pulses per second. This yield can be increased by decrease of the crystal thickness or increase of voltage applied. Thanks to these modifications, the pulse duration can be shortened up to $5 \mathrm{~ns}$.

- Detectors size is extremely small and therefore it can be used for point measurement. In addition, thanks to tiny size, there is almost no impact of the detector on studied system.

- Diamond is a solid form of carbon with a diamond cubic crystal structure. This special structure results in its beauty and in its special properties [7]. The diamond crystal is an unique material, stable in vast chemical environments, and especially it has high radiation hardness [5, 8-10]. Crystal itself is the crucial part of the detector, compared to electrodes, which are not affected by radiation damage. The radiation hardness of the detector is necessary condition for the diamond detectors to be considered as appropriate for such application.

- Charge collection conserves total charge deposited and also the energy deposition location. The type of charge deposition (point or linear) - is reflected in pulse shape. Thanks to this detector property,

\footnotetext{
${ }^{3}$ The First tests [4] contains results of the first VR-1 reactor measurements with the diamond detectors. These experiments were done in 2014 and showed possible application of the diamond detectors for neutron measurements.
}

source particle of interaction can be determined by pulse shape analysis [11 and later can be used for discrimination. Moreover, this allows to count interactions with lower amplitude than gamma background.

On the other hand, the diamond detectors are difficult to manufacture, requires advance technologies for data processing and have several problems that needs to be solved [5, 6. Main detector challenges are:

- The performance of electronics - Signal peak duration is less than 10 ns that results in high requirements on speed of signal and data processing. From our experience, the signal sampling should be higher than $2 \mathrm{Gs} / \mathrm{s}^{4}$ and bandwidth above 250 $\mathrm{MHz}$. For higher impulse rates, the $\mathrm{AD}$ converter needs to work in almost continuous mode without the dead time for data processing.

- The crystal quality - The purity of crystals (low birefringence) is crucial for diamond application. Our work is struggling with impurities in the crystals and with non ideal crystallographic structure. Impurities and dislocation results in decrease of the breakdown voltage. This results in decrease of detector performance and limits the charge collection. Manufacturing of pure diamond crystal underwent vast development during last decades and therefore the diamond crystals can be more used in scientific application. However, still the quality of the electronical grade single crystal diamonds has some fluctuations.

- The quality of the conversion layer and electrode The conversion layers and electrodes are deposited by the physical vapour deposition technology and sometimes the deposited layer laminates or cracks. Thicknesses, materials and deposition technology therefore needs to be further enhanced.

- The neutron flux intensity - Active volume of the crystal is relatively small, and reaching high number of interactions per second can be difficult if the flux is not intense enough [12].

In conclusion, the diamond detectors are promising for precise measurements of neutron flux. However, the diamond detectors for SCRAM measurement that detects thermal neutrons do not exist. Therefore we are forced to develop our own detector.

\subsection{Current Diamond DeVElopment OVERVIEW}

The diamond detector technology is currently under fast development [5, 6. Compared to silicon semiconductors, the diamond technology is delayed due to problems with crystal manufacturing. The process of making artificial diamond is difficult and preparation of clean diamond crystals is much more challenging. Due to that, the diamond detectors are not often used

\footnotetext{
${ }^{4}$ giga samples per second
} 
and are currently being developed. Diamond detectors are mostly used on particle accelerators. Current research activities still include studies of diamond crystal itself (behavior, manufacturing, processing,...). In addition to this, the diamond detector applications are explored and enhanced.

With the improvement of diamond manufacturing technology during last decades, more research is now carried out in field of the diamond detectors. General overview of chemical vapor deposited (CVD) diamond application in radiation detection is presented in [5]. The diamond detectors are developed for variety of application in medicine or accelerators. The diamond based detector with possible application for neutron measurement are currently developed only at CTU in collaboration with CEA Saclay (this work) and TU Vienna under prof. Erich Griesmayer [13].

Griesmayer's research group is not focused at the fast transient measurements but more at the beam applications or spectrum measurements. They are developing diamond based beam monitors, diamond detectors for neutron spectroscopy for fast reactors [14, testing different convectors 13, 15, and more. Their closest detector type to our is the Li converter based detector for thermal neutron measurements [16]. The Griesmayer's research group use the pulse shape analysis for interpretation of measured interactions. They summarize this method here [11, 17, 18].

\section{Proposed DiAmond Detector DESIGN}

The diamond detectors are made of a single crystal electronic grade crystal (ELSC), an electrode material and a conversion layer. Two largest surfaces of the diamond crystal are covered with the super-thin electrode. The conversion layer is placed on the electrode surface. The electrodes and conversion layers are deposited by the physical vapour deposition (PVD). After the deposition, the wires are connected by a special conductive epoxy glue to the electrodes and the detector is ready for measurements. Scheme of this "sandwich" detector design is shown in Figure 1

For the neutron detection - direct and indirect method can be used [19]. Direct method uses direct interaction of neutrons with carbon nuclei [15], but this can be used for fast neutrons only. Thermal neutrons works better with the indirect method, where the converting material is introduced. Several materials 20 22] can be used for neutron conversion to charged particle, which is then detected.

- $B_{4} C$ - The ${ }^{10} B$ isotope is frequently used conversion material for the neutron detection. The boron nuclei absorbs the neutron and emits alpha particle with energy of cca $2 \mathrm{MeV}$. The ${ }^{10} B$ isotope has high cross sections for $\mathrm{n}$ alpha interaction. The alpha particle can be detected in the crystal. The energy is deposited point wisely so it creates signal with specific shape. Based on the shape, the interaction can be identified.

- $\mathrm{Li}$ - The ${ }^{6} \mathrm{Li}$ isotope is frequently used as a conversion material as well. The cross section for the $n$ alpha reaction is around one order smaller than for the ${ }^{10} \mathrm{~B}$. On the other hand, the particle emitted has higher energy.

- Uranium - the neutron induced fission on ${ }^{235} \mathrm{U}$ creates fission fragments that can be detected in the crystal. These fragments carry around $160 \mathrm{MeV}$ and has short range, so they deposit all energy in short distance from the fission interaction. This conversion material creates very high pulses in the detector, however the ${ }^{235} U$ isotope is a nuclear material which requires special attention (nuclear security, proliferation,...).

- Hydrogen - the neutron proton interaction in the hydrogen can be used to produce charged particles, that can interact in the detector volume [15. For our application, it is difficult to place hydrogen on the electrode with sufficient density.

In addition to direct deposition of the conversion material, the material can be also dispersed in the crystal itself (something like the gaseous detectors with $\left.B_{4} F\right)$. This option has problems with a decrease of breakdown voltage and low concentration of the conversion material and therefore it lowers detection efficiency. For our detector, we selected $B_{4} C$ as conversion layer due to several reasons:

- high cross sections for neutron - alpha interaction,

- high reaction rate,

- good mechanical properties, stability,

- possibility to use PVD for deposition,

- vast experience in nuclear field with this conversion material,

- cheap and without restriction.

Another construction part of the detector is the epoxy glue for wire connection. Conductivity of this glue is usually ensured by silver element. However, silver creates as any metal free electrons (from gamma interaction) and based on experience from first measurements [4, is advised to not use metal based glues for the reduction of electron generation. Current market offers the carbon epoxy which can be used instead of silver based epoxies.

\subsection{CONVERSION LAYER THICKNESS OPTIMIZATION}

The simple detector model was created in the MCNP code to calculate the optimal conversion layer thickness. The simple model contain - the crystal, the conversion layer and the neutron source (see Figure 2). Results of this calculation are plotted in Figure 3 As shown, the increase of thickness provides more particles with lower energies. The limit thickness is around 


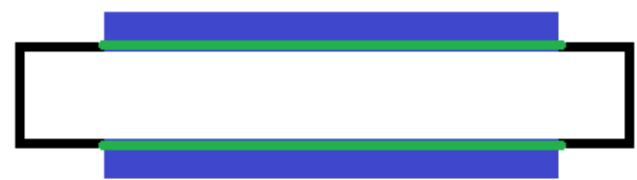

Figure 1. Diamond detector: diamond crystal (white), electrodes (green), conversion layer (blue).

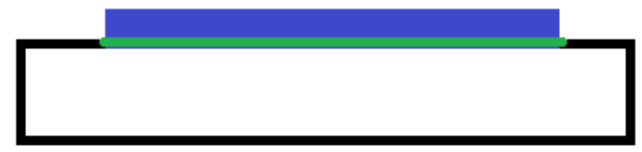

Figure 2. Simple detector model in MCNP (detector - black rectangle, electrode - green, conversion layer blue, neutron source - black dot)

$3.5 \mu \mathrm{m}$. Above this value, no more alpha particles are entering in the diamond crystal.

The optimal thickness is strongly dependent on the data analysis (whether it is possible to discriminate gamma from low energy alphas or not). The pulse shape analysis can distinguish between alpha interactions and other although the alpha particles entering the diamond crystal have low energy. In other words, the thickness can be $3.5 \mu \mathrm{m}$. For the pulse height discrimination, low energy alphas cannot be detected, because the peak height is below the background level. In contrary, the thicker layer is more likely to suffer delamination or have other structural problems. Therefore it is better to use thinner layer. In our detectors, the conversion layers were $1-1.5 \mu \mathrm{m}$ thick.

\subsection{INTERACTIONS IN THE DETECTOR}

All charged particles interact in the diamond detector, however, they differ in the energy deposition method. The alpha particle deposits its energy point vise (Bragg Peak 21]), compared to gamma and elec-

\begin{tabular}{cc}
\hline particle & signal \\
\hline alpha & square pulse \\
\hline electrons, gamma & triangular pulse \\
\hline neutron & mixed pulse \\
\hline
\end{tabular}

TABLE 1. Signal shape for different particles

trons which use the linear deposition. This difference is useful in separation of neutron signals from others. The energy deposited by alpha interaction is too low, so these peaks are mixed in other interactions, and therefore the pulse height discrimination is not applicable. On the other hand, the pulse shape separation can be used thanks to the different energy deposition method.

For this application, the neutron detection is crucial. Neutrons are commonly detected in this detector indirectly. At first, the neutron interacts with boron in the conversion layer. The neutron capture results in the alpha particle emission. Then the alpha particle interacts with the diamond crystal, depositing its energy to holes and free electrons. Due to electrical field introduced by high voltage applied on electrodes, holes and electrons drifts to electrodes and charge is collected. Usually $100 \mathrm{~V}$ per $100 \mu \mathrm{m}$ is used. Collected charge creates typical rectangular pulse [11]. The peak height can be adjusted by high voltage change. If higher voltage is applied, the charge is collected faster and therefore the peak duration is shorter and the peak height is increased. Neutron can also (rarely) interact directly in the crystal [15], with different signal shape. For this application proposed, direct interactions are not important.

Compared to previous cases, electron and gamma particles interact in the diamond crystal differently. The energy is deposited linearly on the whole path of the traversing particle. This results in different ionization and therefore the peak has different - triangular - shape [1]. For both interactions, pulse area corresponds to absolute value of deposited energy. Pulse shape depends on type of energy deposition and can be used for the interaction separation [11. Particles and their interactions are noted in the Table 1

The difference between shapes created by the alpha and the electron interaction in the crystal are shown in Figure 4. The cases 1) and 2) show the alpha interaction in different crystal areas. On the right side of the figure, the theoretical shapes produced by the interaction are displayed. The case 3 ) represents the electron or gamma energy deposition in the crystal and its pulse shape. As it was mentioned previously, the direct neutron interaction creates something like stair shaped pulse.

\subsection{Pulse shape method implementation}

After the interaction, the charge is collected by electrical field. The detector produces pulses with duration 


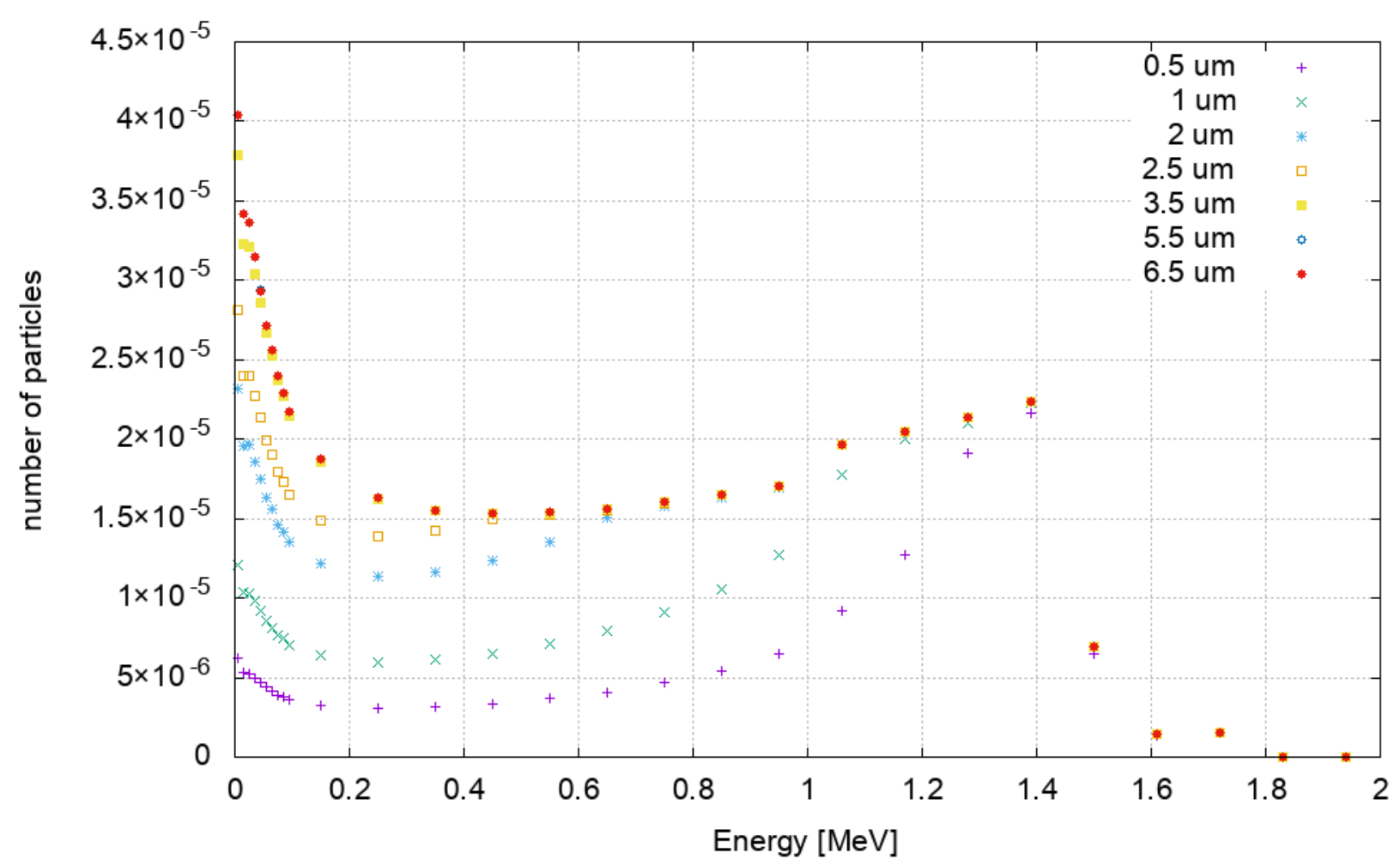

FiguRE 3. Energy spectrum on border of the crystal for different conversion layer thicknesses

1)

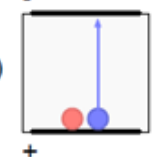

2)

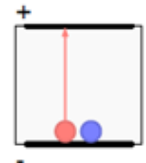

3)

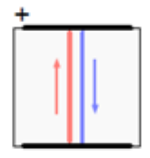

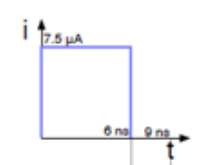
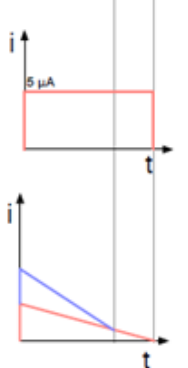
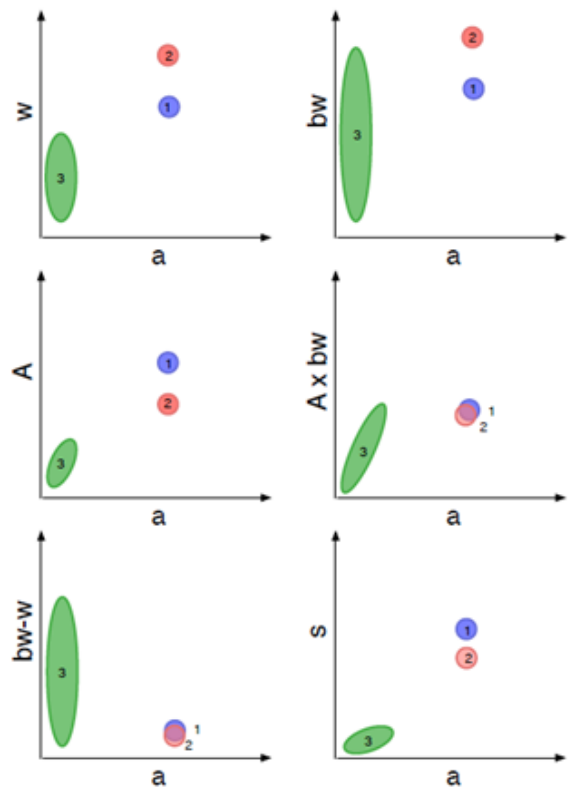

FiguRE 4. Theoretical interaction of 1) and 2) alpha particles; 3) gamma or electrons [11] (a - area, A - amplitude, w - width, bw - base width) 
$8 \nu s$ (deepens on applied voltage and diamond thickness) approximately. The signal from the detector is firstly amplified by the charge sensitive amplifier. Then the whole pulse is recorded for the pulse shape analysis. The AD converter should have high bandwidth and high sampling rate so the the shape of signal is preserved. Our recommendation is to have bandwidth above $250 \mathrm{MHz}$ and sampling above 2 GS/s. For recording purposes, the pulse is identified by pulse-height separation (only for the basic noise signal separation) and values are recorded. 5 Recorded pulses are later processed by the Matlab script (developed in collaboration with CEA, based on [1]) which characterize each pulse by several parameters:

- Peak height

- Mid Height Width - this is FWHM equivalent

- Drift time

- Qtot - total deposited energy

- Form factor - this special coefficient compares the calculated area vs the measured area. The calculated area is a rectangular shaped (Peak height multiplied by peak length). If values of calculated and measured area are close, pulse shape should be rectangular as well (see Figure 5].

- Qtail - area (energy) of the peak tail part (after peak maximum)

- Qtail vs Qtot - tail vs total area

The processing Matlab script produces two output files. One text file, with above mentioned parameters for each pulse, and one plot file with plotted results (see Figure 6). For demonstration, data from an alpha source measurement (including gamma and background) were processed by the script and are presented in the Figure 6. Alpha interactions cannot be separated by the pulse height method only (commonly used discrimination method). As it is possible to see in the upper second chart, interactions from alpha particles includes all pulse heights (marked by green). Some of them are higher than other pulses from different interactions, but most of them has similar peak height. This means, that pulse height separation will result in much lower yield than possible. However, as it was described previously, the alpha interactions can be separated by their shape using the form factor and the Mid Height Width parameter. The orange circle in Figure 6 marks all alpha interactions (low form factor and similar Mid Height width). Form factor needs to be close to 1 and Mid Height Width is around $6 \nu s$ in this case. As shown, by simple conditioning of the form factor value, the alpha interactions can be separated from other signals. Combination of other parameters can be used to enhance the separation.

\footnotetext{
${ }^{5}$ In our case we use the DT5743 8 Channel 12bit 3.2 GS/s Switched Capacitor Digitizer (details at CAEN web page [23]).
}

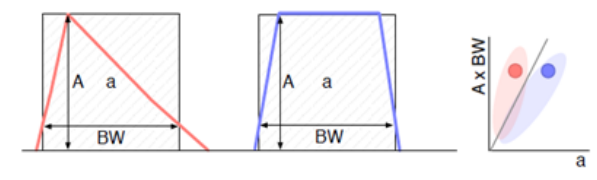

Figure 5. The form factor schematic 11]

\section{DiAmond DETECTOR MANUFACTURING}

After large theoretical studies and simulations, three detectors were manufactured in CEA Saclay from one diamond crystal (EL SC Plat $\mathrm{f}^{6} 3.0 \times 3.0 \mathrm{~mm}, 0.50 \mathrm{~mm}$ thick). During manufacturing and testing several problems occurred, some of them were successfully solved, some still needs more research effort. Design parameters of manufactured detectors are noted in Table 2, Detailed description of the detector manufacturing, including problem faced is presented in the following sections.

\subsection{THE FIRST DETECTOR}

\begin{tabular}{cc}
\hline Parameter & Value \\
\hline diamond thickness & $0.5 \mathrm{~mm}$ \\
\hline Diamond size & $3 \mathrm{~mm} \mathrm{x} 3 \mathrm{~mm}$ \\
\hline Electrode material & Aluminum \\
\hline conversion layer material & B4C \\
\hline
\end{tabular}

TABLE 2. Parameters of the diamond detector

The first detector was manufactured after the crystal inspection and cleaning. Two layers of material were deposited on each side of the diamond crystal by the technique of Physical Vapour Deposition. The first, very thin layer of conductive metal (aluminum) serves as the electrode. The second, boron layer serves as the conversion layer that uses the $\mathrm{n}$ - alpha interaction to convert non charged particles (neutrons) to charged particles (alpha). Both layers are shown in Figures 7 and 8. Electrodes were round shaped and the conversion layers were square shaped. Probably, due to higher thickens of the second conversion layer, several cracks appeared (see Figure 8). However, these structural problems have no influence on the conversion itself.

After the electrode deposition, the wires were connected to the electrodes by the conductive resin. In this case, the silver resin was used. For the other detectors, we used the conductive carbon epoxy (to decrease gamma - electron interaction). The first detector with wires connected is shown in Figure 9 .

\footnotetext{
${ }^{6}$ electronic grade single crystal, The diamond crystal was manufactured by the Element six company.
} 


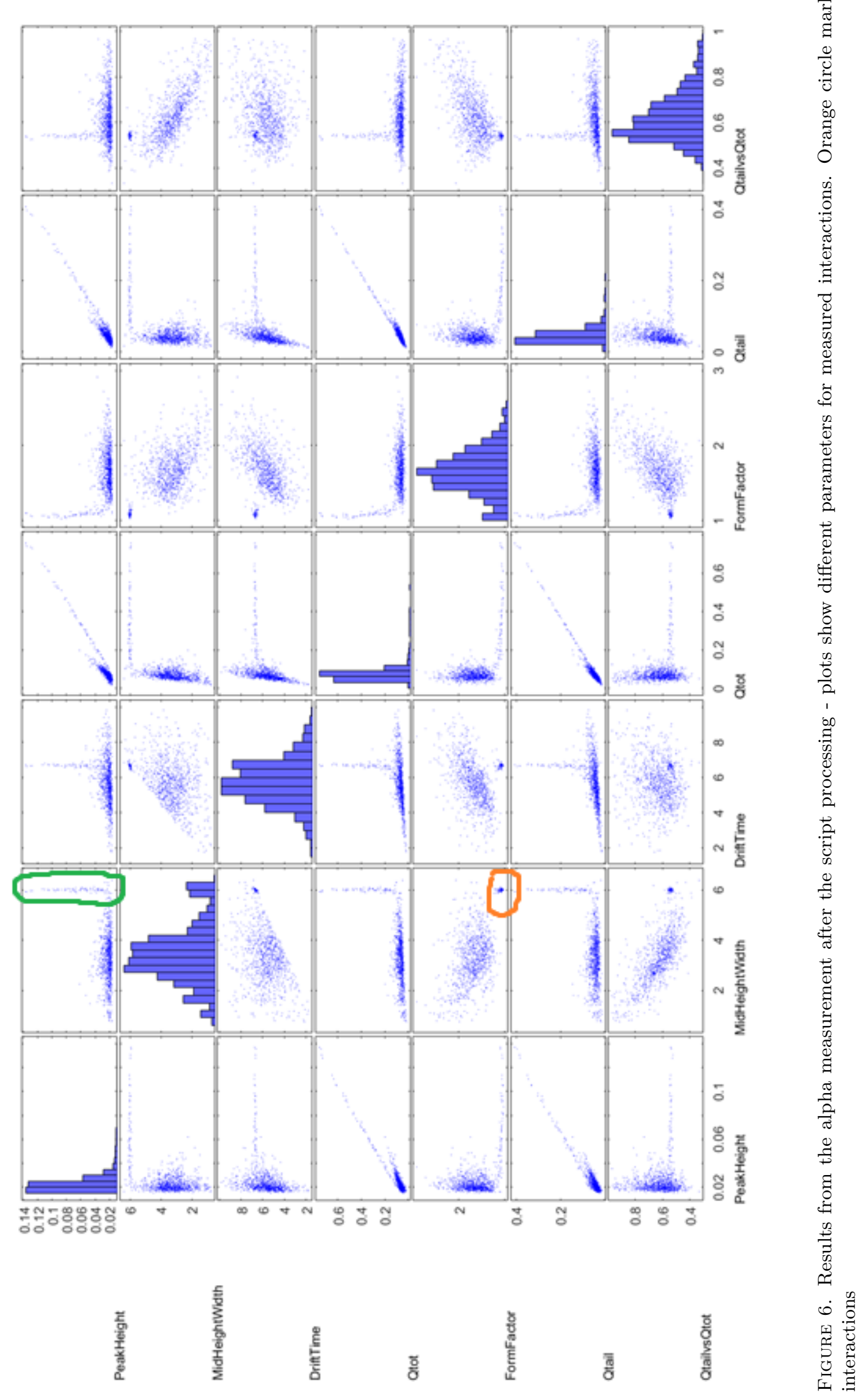




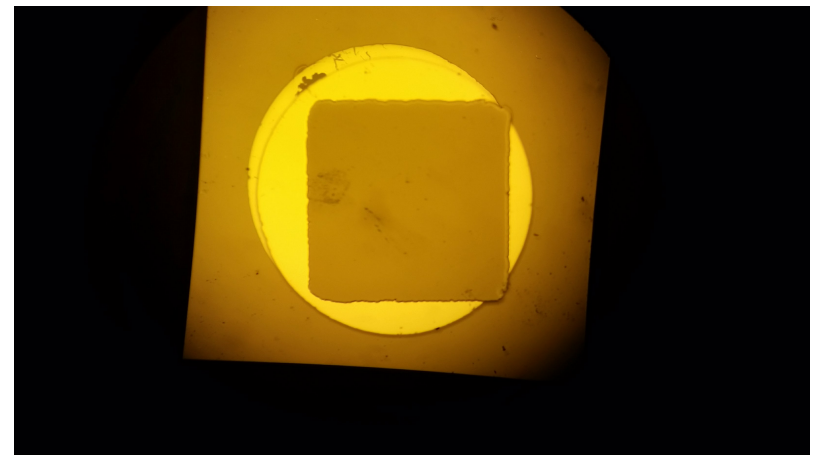

Figure 7. The electrode and the conversion layer at diamond crystal - first side (first detector)

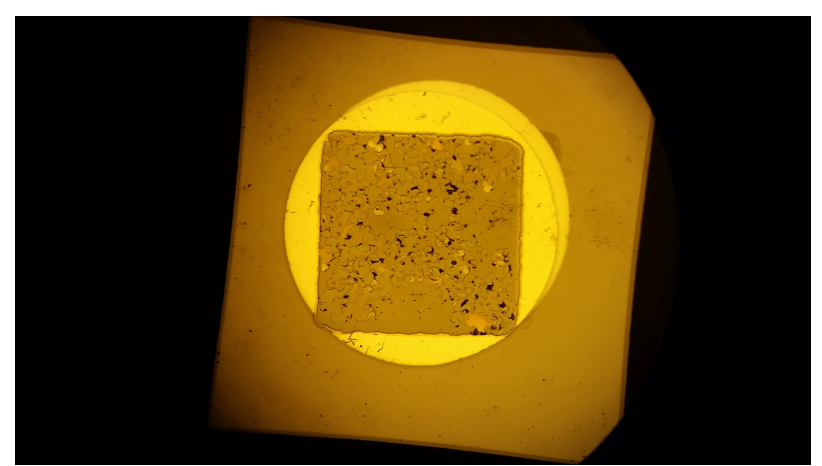

Figure 8. The electrode and the conversion layer at the diamond crystal - second side (first detector)

For the detector mechanical protection, the first detector was sealed in a small flask filled with a nonconductive epoxy. The detector was then installed in a plastic box and connected to the BNC connector as shown in the Figure 10

After several measurements with the first detector, the bridge current occurred. This means, when the high voltage (up to $500 \mathrm{~V}$ ) was applied, the current started to flow through the detector. The breakpoint was somewhere above $50 \mathrm{~V}$. Sometimes higher, sometimes lower. Our first idea was that the current is caused by some structural problems in the crystal (probably some dislocations). Therefore to repair the detector, we tried to heat the detector up to $70{ }^{\circ} \mathrm{C}$ (to remove the dislocations from crystal) 7 This method did not help and thus the first detector was dismantled, the crystal was cleaned and used for a new detector.

We expected, that the conductive connection is somewhere over the edge of the crystal (probably some impurities in the non conductive epoxy made conductive connection). This problem should be solved by cleaning. Our other idea was that structural problems of the crystal resulted in Bridger current and the previous treatment $\left(70{ }^{\circ} \mathrm{C}\right)$ was not successful. However, during the cleaning process, the crystal is boiled in acid for long time period. This high temperature treatment can repair the crystal.

\footnotetext{
${ }^{7}$ The temperature $70{ }^{\circ} \mathrm{C}$ was the highest possible temperature that does not destroy the detector.
}

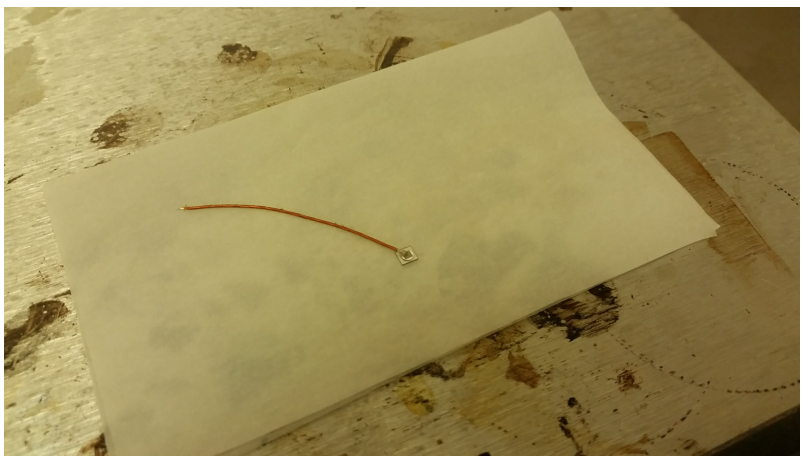

Figure 9. The diamond detector with wire glued to the electrode by silver resin (first detector)

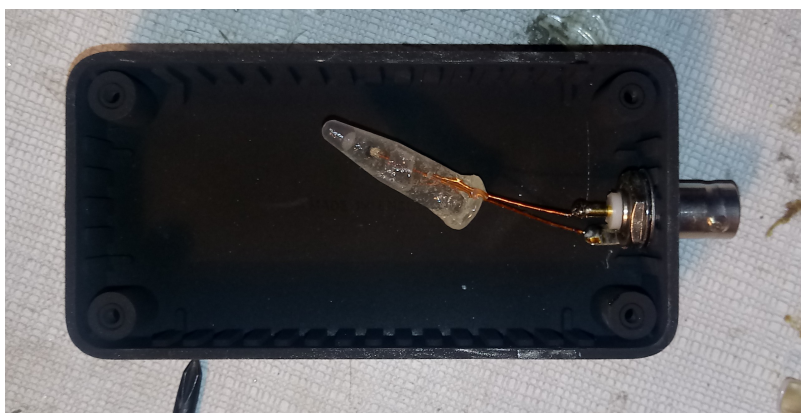

Figure 10. The detector inside the boron plastic box (the first detector)

\subsection{The SECOND DETECTOR}

The second detector was produced in the same way as the first one, except the epoxy for wire connection. Instead of the silver conductive epoxy, the carbon conductive epoxy was used. The deposition of two $B_{4} C$ layers was done as in previous case and did not show any structural problems right after the deposition. However, after weekend, one of the $B_{4} C$ layers was cracked and started to delaminate (see Figure 11). The second layer was without structural problems. We were unable to find the reason for this delamination. Under the polarized light, it was possible to observe, that the aluminum electrode delaminated with the $B_{4} C$ layer together, this means that the delamination occurred between the diamond surface and the aluminum layer. To solve this problem and finalize the detector, we removed the delaminated area, cleaned the diamond surface with the plasma cleaner and deposited only the aluminum electrode. At the end, we have received a crystal with the aluminum electrode on both surfaces and one B4C layer. The conversion layer measured around $1 \mu \mathrm{m}$ in thickness. Then the wires were connected to the aluminum electrodes by the carbon conductive epoxy. Sadly, the current measurement showed that the detector is not functional, due to the current going through the detector. The root of this current was probably in the carbon epoxy bridge over the edge of the crystal. The process of placing small drops to the crystal surface was not properly done and resulted in the bridge conductive connection of both electrodes. The electrodes were 


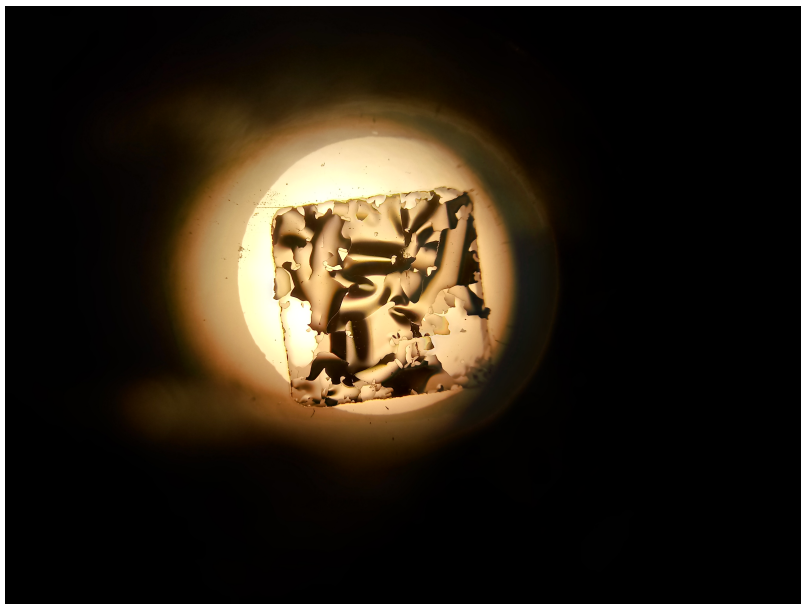

Figure 11. Delaminating layer on the second detector

probably connected over the edge of the diamond crystal. To solve this problem, we improved the consistency of the carbon epoxy and made several tests of different syringes and pipets to enhance the dropping technique. However, the detector was not functional and therefore it was dismantled again.

\subsection{THE THIRD DETECTOR}

Based on previous experience, the third version of the detector was created in the same way as the previous one. This detector has one $\mathrm{B} 4 \mathrm{C}$ conversion layer, approximately $1.5 \mathrm{um}$ thick. Wires are connected to the electrode by the carbon conductive epoxy. The detector was tested and had no leakage current and no bridge current.

In the end, the crystal with wires is closed in a plastic flask (see Figure 12 Compared to the first detector, the flask is not filled with the epoxy but contains air. The flask itself is glued to the box. This third detector was tested under large natural uranium source and showed expected responses to incoming radiation.

\section{Conclusion}

The time dependent measurement of neutron flux distribution changes during the SCRAM is highly challenging and requires high performance from the detector system. However, the current detectors available at the market are not capable of such measurements. Therefore, this paper presents the progress in the diamond detector development, aimed to the fast neutron flux change measurements in a nuclear reactor introduced by the SCRAM.

The diamond detector is based on the Electrical grade single crystal with a boron conversion layer and aluminum electrodes. This configuration allows to detect thermal neutrons by the neutron - alpha conversion in the boron layer. The alpha particle later interacts in the diamond crystal and deposits the energy. High voltage applied on the diamond detector

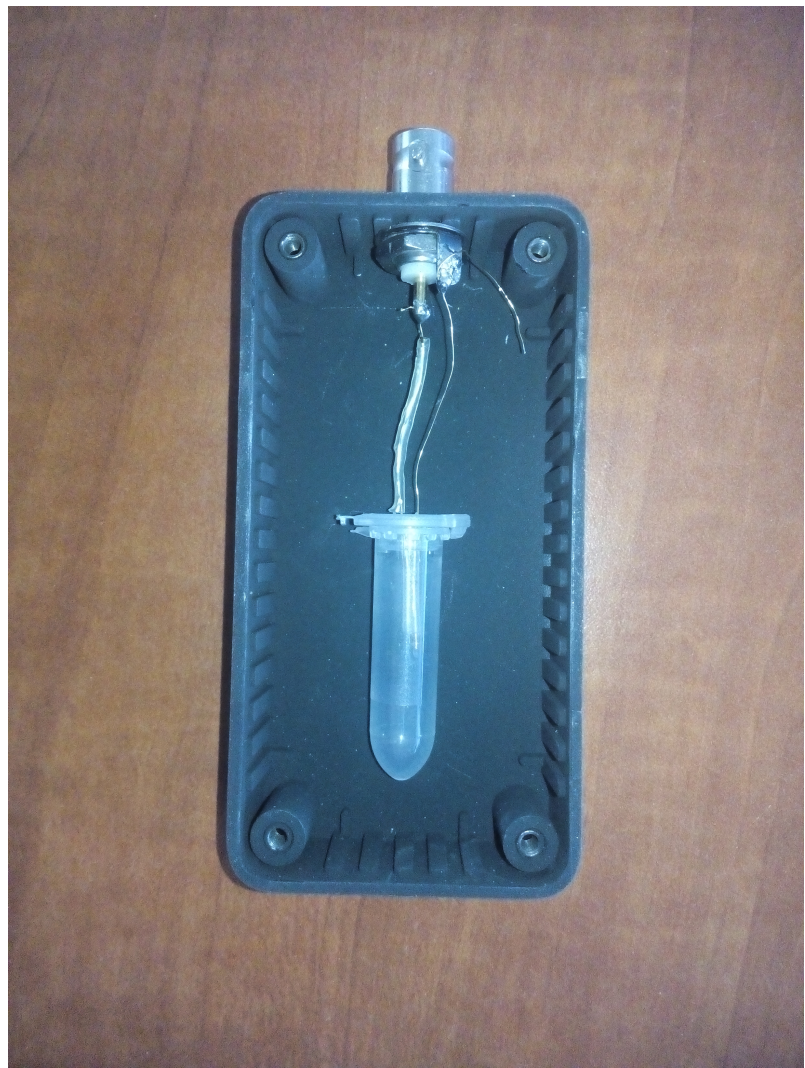

Figure 12. The detector inside the boron plastic box (the third detector)

collects the charge. All pulses are recorded including gamma, electron and direct neutron interactions. Neutron interactions are selected by the pulse shape analysis.

To prepare the preliminary detector design, the current experience in the field of the diamond detector was studied. The first part of this paper presents overview of work in the diamond detector field with special attention to neutron flux measurements. Theoretical study was combined with several MCNP calculations to prepare the design of the detector. The MCNP calculations were performed to understand the detector performance and to optimize the main detector parameters (e.g. conversion layer thickness). Obtained results are presented for example in Figure 3 Detector design was used in three experimental detectors, that were prepared in CEA Saclay. As a main part of the study presented, especially the manufacturing process and detector design parameters were developed and enhanced. During this work, several problems arised (breakdown currents, conversion layer delamination,...). Some problems were solved. These challenges are more described in section 4 For the purpose of the gamma background discrimination, the pulse shape analysis was selected and implemented in the data processing. The MATLAB script was prepared for data processing. Description of the detector interactions and the pulse shape analysis is presented in section 3.2 . The first results of a simple test mea- 
surement with the alpha source are presented in Figure 6. In the example presented, the location of alpha interaction is marked. In this case, the discrimination conditions were not stated. Detail experimental work will examine and optimize the discrimination conditions (results will be published in the next paper).

The progress achieved in the detector development is promising. This work will be followed by experimental testing with various radiation and neutron sources to study detector performance and to enhance the pulse shape analysis. The optimization of discrimination parameters should be performed before the detector will be tested in the VR-1 nuclear reactor, which will be another step of this SCRAM study.

\section{ACKNOWLEDGEMENTS}

Support from IAEA fellowship number C6/CZR/17003 was used in this work. This work was supported by the Grant Agency of the Czech Technical University in Prague, grant No. SGS18/146/OHK4/2T/14.

\section{REFERENCES}

[1] International atomic energy agency. Safety of Research Reactors. IAEA Safety Standards IAEA, Vienna 2016.

[2] L. Sklenka. Reactor Physics Experiments at Low Power Research Reactors. Habilitation Thesis Czech Technical University in Prague, Prague, 2013.

[3] T. Bily, et al. Effect of kinetics parameters on transients calculations in external source driven subcritical VR-1 reactor. Annals of Nuclear Energy pp. 97-109, 2019. ISSN 0306-4549.

[4] M. Pomorski. Diamond as a solid state micro-fission chamber for thermal neutron detection. Talks presented at the 3rd ADAMAS Collaboration Meeting ECT*, Trento November 18 - November 20, 2014, available at http://www-adamas.gsi.de/ADAMAS03/talks/ Pomorski_CEA.pdf (accessed on January 15st, 2018).

[5] P. Bergonzo, A. Brambilla, D. Tromson, et al. CVD diamond for nuclear detection applications. Nuclear Instruments and Methods in Physics Research Section A: Accelerators, Spectrometers, Detectors and Associated Equipment pp. 694-700, 2002. ISBN 0168-9002. ISSN 0168-9002, DOI:10.1016/S0168-9002(01)01668-0.

[6] R. Sussmann. CVD Diamond for Electronic Devices and Sensors. John Wiley ES Sons 2009. ISBN 9780470065327, DOI:10.1002/9780470740392.

[7] H. Yang, J. Xiao, Z. Yao, et al. Homogeneous and heterogeneous dislocation nucleation in diamond. Diamond and Related Materials 88 pp. 110-117, 2018. ISBN 0925-9635, DOI:10.1016/j.diamond.2018.07.005

[8] C. Bauer, et al. Radiation hardness studies of CVD diamond detectors. Nuclear Instruments and Methods in Physics Research Section A: Accelerators, Spectrometers, Detectors and Associated Equipment,367,1-3 pp. 207211, 1995. Available at: https://www.sciencedirect. com/science/article/pii/0168900295005455.

[9] A. Metcalfe, et al. Diamond based detectors for high temperature, high radiation environments. Journal of Instrumentation 1201 2017. C01066, ISBN 1748-0221, DOI:10.1088/1748-0221/12/01/C01066
[10] Y. Sato, et al. Radiation hardness of a single crystal CVD diamond detector for $\mathrm{MeV}$ energy protons. Nuclear Instruments and Methods in Physics Research Section A: Accelerators, Spectrometers, Detectors and Associated Equipment 784 pp. 147-150, 2015. DOI:10.1016/j.nima.2014.12.036

[11] M. Cerv. CVD diamond application for particle detection and identification in high radiation environments. PhD thesis Technische Universitat Wien Supervised by Univ Doz Dipl-Ing Dr Erich Griesmayer, Wien 2016.

[12] J. Rataj, et al. Benchmark experiments for validation of reaction rates determination in reactor dosimetry. Radiation Physics and Chemistry 104 pp. 363-367, 2014. ISSN 0969-806X.

[13] E. Griesmayer. CIVIDEC Instrumentation. 6th ADAMAS Collaboration Meeting@ECT 2017. Trento, Available at: http://www-adamas.gsi.de/ADAMAS06/ talks/Griesmayer_2017.pdf

[14] M. Osipenko. Neutron spectrometer based on diamond detectors for fast reactors. 6th ADAMAS Collaboration Meeting @ ECT 2017. Trento, Available at: http://www-adamas.gsi.de/ADAMAS06/talks/ Osipenko_2017.pdf

[15] C. Weiss. Neutron Application with Diamond Detectors. 6th ADAMAS Collaboration Meeting ECT Trento, 2017, Available at: http://www-adamas.gsi. de/ADAMAS06/talks/Weiss_2017.pdf.

[16] M. Hursin, et al. Testing of a sCVD diamond detection system in the CROCUS reactor. The European Physical Journal A 545 82, 2018. ISSN 1434-6001, DOI:10.1140/epja/i2018-12519-1.

[17] P. Kavrigin, et al. Pulse-shape analysis for gamma background rejection in thermal neutron radiation using CVD diamond detectors. Nuclear Instruments and Methods in Physics Research Section A: Accelerators, Spectrometers, Detectors and Associated Equipment 795 pp. 88-91, 2015. ISBN 0168-9002, DOI:10.1016/j.nima.2015.05.040

[18] C. Weiss, et al. Selective data analysis for diamond detectors in neutron fields. EPJ Web of Conferences 146, 2017. EDP Sciences,ISBN 2100-014X, DOI:10.1051/epjconf/201714603004.

[19] J. Rataj, et al. Reactor physics course at VR-1 reactor 2nd ed. Prague: Czech Technical University in Prague, 2014, ISBN 9788001055014.

[20] T. Crane, M. Baker. Neutron detectors 1997. Available at: http:

//www.lanl.gov/orgs/n/n1/panda/00326408.pdf (accessed on January 21st, 2018).

[21] G. Knoll. Radiation detection and measurement Hoboken, N.J.: John Wiley, c2010, ISBN 0470131489.

[22] F. Thevenot. Boron carbide - a comprehensive review. Journal of the European Ceramic society $64 \mathrm{pp}$. 205-225, 1990. ISSN 0955-2219, DOI:10.1016/0955-2219(90)90048-K.

[23] DT5743. 8 Channel 12bit 3.2 GS/s Switched Capacitor Digitizer Caen, (accessed on July 16th, 2018) Available at: http://www.caen.it/csite/CaenProd. jsp?parent=14\&idmod=823. 